\title{
The Influence of a Magnetized Plasma Column on the Radiation Characteristics of a Strip Loop Antenna
}

\author{
Alexander V. Kudrin ${ }^{1}$, Anna S. Zaitseva ${ }^{2}$, Bernardo Spagnolo ${ }^{3}$, Tatyana M. Zaboronkova ${ }^{4}$ \\ ${ }^{1}$ Dept. of Radiophysics, University of Nizhny Novgorod, Nizhny Novgorod, Russia, Email: kud@ rf.unn.ru \\ ${ }^{2}$ Dept. of Radiophysics, University of Nizhny Novgorod, Nizhny Novgorod, Russia, Email: anaze@yandex.ru \\ ${ }^{3}$ Dept. of Physics and Chemistry, Palermo University, Palermo, Italy, Email: bernardo.spagnolo@unipa.it \\ ${ }^{4}$ Dept. of Nuclear Physics, R. E. Alekseev Nizhny Novgorod State Technical University, \\ Nizhny Novgorod, Russia, Email: zabr@nirfi.sci-nnov.ru
}

\begin{abstract}
The radiation characteristics of a circular loop antenna located on the surface of an open waveguide in the form of an axially magnetized plasma column are studied using the rigorously obtained current distribution of such an antenna. The radiation resistance of the antenna excited by a time-harmonic external voltage is obtained for the case where the plasma inside the column is resonant. The distribution of the radiated power over the spatial spectrum of the excited waves is found and discussed.
\end{abstract}

Index Terms-magnetized plasma column, loop antenna, radiated power, radiation resistance.

\section{INTRODUCTION}

The characteristics of loop antennas immersed in a magnetoplasma have been studied extensively in many works (see, e.g., [1] and references therein). In the past decade, enhanced interest has been shown in the characteristics of antennas operated in the presence of magnetic-field-aligned cylindrical plasma structures capable of guiding the excited electromagnetic waves [2]. Recently, the current distribution and input impedance of a strip loop antenna located on the surface of a plasma column have been studied in the case where the column is filled with a resonant magnetoplasma [3]. In this work, we extend the analysis of [3] to consideration of the radiation characteristics of such an antenna. Note that by resonant magnetoplasma, we mean a cold, collisionless magnetized plasma in which the refractive index of one of the characteristic waves tends to infinity at a certain angle between the wave vector and an external dc magnetic field [2]. It should be noted that the column filled with a resonant magnetoplasma supports the guided propagation of an infinite number of eigenmodes including the quasielectrostatic modes [4]. Therefore, the spatial spectrum of the antennaexcited waves consists of both continuous- and discretespectrum contributions. Moreover, the presence of an infinite number of eigenmodes appreciably affects the behavior of the antenna electrodynamic characteristics [3].

\section{Formulation of the Problem}

Consider an antenna having the form of an infinitesimally thin, perfectly conducting narrow strip of half-width $d$ coiled into a circular loop of radius $a(d \ll a)$. The antenna is located coaxially on the surface of a uniform circular plasma column surrounded by a homogeneous isotropic medium with the real dielectric permittivity $\varepsilon_{\text {out }}=\epsilon_{0} \varepsilon_{a}$, where $\epsilon_{0}$ is the permittivity of free space. The column is aligned with an external dc magnetic field $\boldsymbol{B}_{\mathbf{0}}$ (see Fig. 1) which is parallel to the $z$ axis of a cylindrical coordinate system $(\rho, \phi, z)$. The medium inside the column is assumed to be a cold collisionless plasma described by a general dielectric tensor with the following nonzero elements: $\varepsilon_{\rho \rho}=\varepsilon_{\phi \phi}=\epsilon_{0} \varepsilon, \varepsilon_{\rho \phi}=-\varepsilon_{\phi \rho}=-i \epsilon_{0} g$, and $\varepsilon_{z z}=\epsilon_{0} \eta$. The elements $\varepsilon, g$, and $\eta$ of the plasma dielectric tensor are functions of the angular frequency $\omega$, and expressions for them can be found elsewhere [2]. Recall that for a resonant magnetoplasma the condition $\operatorname{sgn} \varepsilon \neq \operatorname{sgn} \eta$ holds for the diagonal tensor elements.

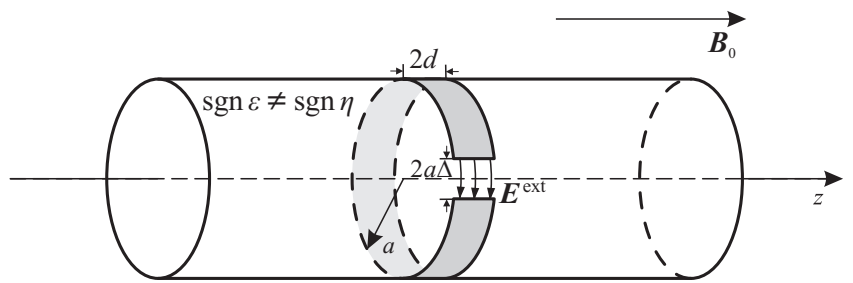

Fig. 1. Geometry of the problem.

The antenna is excited by a time-harmonic $(\sim \exp (i \omega t))$ voltage which creates an electric field with the only nonzero component $E_{\phi}^{\text {ext }}$ in a narrow angular interval $\left|\phi-\phi_{0}\right| \leq \Delta \ll$ $\pi$ on the surface of the strip (i.e., at $\rho=a$ and $|z|<d$ ):

$$
\begin{aligned}
E_{\phi}^{\mathrm{ext}}(a, \phi, z)= & \frac{V_{0}}{2 a \Delta}\left[U\left(\phi-\phi_{0}+\Delta\right)-U\left(\phi-\phi_{0}-\Delta\right)\right] \\
& \times[U(z+d)-U(z-d)]
\end{aligned}
$$

Here, $V_{0}$ is an amplitude of the given voltage, $U$ is a Heaviside 
function, and $\Delta$ is the angular half-width of the gap centered at $\phi=\phi_{0}$. The excitation field $E_{\phi}^{\text {ext }}$ can be represented as

$$
E_{\phi}^{\mathrm{ext}}=\frac{V_{0}}{2 \pi a} \sum_{m=-\infty}^{\infty} \frac{\sin (m \Delta)}{m \Delta} \exp \left[-i m\left(\phi-\phi_{0}\right)\right] .
$$

The surface density $I(\phi, z)$ of the electric current excited on the antenna by field (1) admits the analogous representation:

$$
I(\phi, z)=\sum_{m=-\infty}^{\infty} \mathcal{I}_{m}(z) \exp (-i m \phi)
$$

As is shown in [3], it is possible to obtain integral equations for the quantities $\mathcal{I}_{m}(z)$ using the boundary conditions on the surface of the plasma column and on the antenna surface and then reduce the problem of the antenna current distribution to solving the corresponding integral equations (see [3] for details). The total current $I_{\Sigma}(\phi)$ is yielded by integrating the surface current density $I(\phi, z)$ across the strip conductor of the antenna. As a result, the rigorous solution for the current distribution $I_{\Sigma}(\phi)$ of the above-described antenna in the case of a resonant magnetoplasma in the column has the following form [3]:

$$
I_{\Sigma}(\phi)=\frac{i V_{0}}{Z_{0} k_{0} a} \sum_{m=-\infty}^{\infty} \frac{\sin (m \Delta)}{m \Delta} \frac{\delta_{m} \exp \left[-i m\left(\phi-\phi_{0}\right)\right]}{\ln (4 a / d)-S_{m}},
$$

where

$$
\delta_{m}=-\frac{i\left(k_{0} a\right)^{2} \xi}{m^{2}+i\left(k_{0} a\right)^{2} \xi}, \quad \xi=\frac{|\varepsilon \eta|^{1 / 2}+i \varepsilon_{a}}{2},
$$

$Z_{0}$ is the impedance of free space, $k_{0}=\omega / c$ is the wave number in free space, and the coefficients $S_{m}$ are determined by the regular (nonsingular) parts of kernels of the integral equations for $\mathcal{I}_{m}(z)$. Expressions for these coefficients can be found in [3]. Upon calculation of $I_{\Sigma}(\phi)$, one can obtain all electrodynamic characteristics of the antenna, including its radiated power and radiation resistance.

\section{Power RAdiATED}

The total power radiated from the antenna with the found current (4) in the presence of a cylindrical plasma column immersed in an isotropic medium can be obtained using standard techniques [4]. With allowance for the guided eigenmodes, an expression for the total radiated power takes the form

$$
P_{\Sigma}=P^{\mathrm{cs}}+\sum_{m, \pm n} P_{m, \pm n}^{\mathrm{mod}}
$$

where $P^{\mathrm{cs}}$ is the power radiated to the continuous-spectrum waves and $P_{m, \pm n}^{\bmod }$ is the power going to an eigenmode with the indices $m$ and $\pm n$. Here, the signs " + " and "-" of the radial index $n$ denote the eigenmodes propagating away from the source region in the positive and negative directions of the $z$ axis, respectively. The power $P^{\mathrm{cs}}$ going to the continuousspectrum waves in the loss-free case is the power radiated to the surrounding medium $P_{\text {rad }}$. This quantity can be found by integrating the time-averaged Poynting vector over the surface of an infinitely long cylinder of radius $\rho=\rho_{a} \geq a$ :

$P_{\text {rad }}=\frac{1}{2} \int_{-\infty}^{\infty} \int_{0}^{2 \pi} \operatorname{Re}\left[E_{\phi}(\boldsymbol{r}) H_{z}^{*}(\boldsymbol{r})-E_{z}(\boldsymbol{r}) H_{\phi}^{*}(\boldsymbol{r})\right] \rho d \phi d z$.

Here, the asterisk denotes complex conjugation, and $E_{\phi, z}(\boldsymbol{r})$ and $H_{\phi, z}(\boldsymbol{r})$ are the azimuthal and longitudinal components of the electromagnetic field excited by the antenna with current $I_{\Sigma}(\phi)$.

An expression for the partial power $P_{m, n}^{\bmod }$ going to an eigenmode with the indices $m$ and $n$ can be derived by integrating the time-averaged Poynting vector for this mode over the cross section $z=$ const $>0$. This formula has the following form:

$$
\begin{aligned}
P_{m, n}^{\bmod }= & \frac{1}{2} \int_{0}^{\infty} \int_{0}^{2 \pi} \operatorname{Re}\left[E_{\rho ; m, n}(\boldsymbol{r}) H_{\phi ; m, n}^{*}(\boldsymbol{r})\right. \\
& \left.-E_{\phi ; m, n}(\boldsymbol{r}) H_{\rho ; m, n}^{*}(\boldsymbol{r})\right] \rho d \phi d \rho,
\end{aligned}
$$

where the quantities under the integral signs are the transverse (with respect to the $z$ axis) components of the electromagnetic field $\left(\boldsymbol{E}_{m, n}, \boldsymbol{H}_{m, n}\right)$ of the corresponding eigenmode $[2,4]$. The total power $P^{\text {mod }}$ going to all eigenmodes, i.e., discretespectrum waves, is determined as

$$
P^{\mathrm{mod}}=\sum_{m, \pm n} P_{m, \pm n}^{\mathrm{mod}}=\sum_{m=-\infty}^{\infty} \sum_{n=1}^{\infty} 2 P_{m, n}^{\mathrm{mod}} .
$$

When deriving Eq. (9), we took into account the symmetry of the problem over the plane $z=0$, which allows us to calculate the contribution of the eigenmodes propagating from the source region in the positive direction of the $z$ axis and then double the quantities $P_{m, n}^{\text {mod }}$ in the resulting formula (9).

The above results allow us to write the total radiation resistance as

$$
R=\frac{2 P_{\Sigma}}{\left|I_{\Sigma}\left(\phi_{0}\right)\right|^{2}}=R^{\mathrm{cs}}+\sum_{m=-\infty}^{\infty} \sum_{n=1}^{\infty} 2 R_{m, n}^{\mathrm{mod}},
$$

where $R^{\mathrm{cs}}$ and $R_{m, n}^{\mathrm{mod}}$ are the partial radiation resistances to the continuous-spectrum waves and eigenmodes, respectively.

\section{NUMERICAL RESUlTS}

Using the above-described approach, we have calculated the total radiated power and the partial radiation resistances $R^{\mathrm{cs}}$ and $R_{m, n}^{\bmod }$ of the antenna. Calculations have been performed for the following values of the parameters: the electron plasma frequency $\omega_{\mathrm{p}}=1.78 \times 10^{11} \mathrm{~s}^{-1}$ (the plasma density inside the column is equal to $\left.N=10^{13} \mathrm{~cm}^{-3}\right)$, the electron gyrofrequency $\omega_{\mathrm{H}}=1.41 \times 10^{10} \mathrm{~s}^{-1}$ (the external dc magnetic field $B_{0}=800 \mathrm{G}$ ), the relative dielectric permittivity of the background medium is equal to $\varepsilon_{a}=1$ (free space), and the angular frequency $\omega=1.7 \times 10^{8} \mathrm{~s}^{-1}$. The chosen values can easily be realized under laboratory conditions and correspond to the case of a resonant plasma inside the column $\left(\varepsilon=1.62 \times 10^{2}\right.$ and $\left.\eta=-1.1 \times 10^{6}\right)$. It is assumed that the midpoint of the region to which the given voltage is supplied 
has the azimuthal coordinate $\phi_{0}=0$, the antenna radius $a$ coinciding with the column radius is equal to $a=2.5 \mathrm{~cm}$, $d / a=0.02$, and $\Delta=0.05 \mathrm{rad}$.

Calculations show that in the case considered, where the inequality $|\varepsilon \eta| \gg \varepsilon_{a}^{2}$ holds, the antenna radiation resistance $R$ is almost completely determined by the contribution of eigenmodes. Therefore, we briefly discuss the dispersion properties and field structures of the eigenmodes guided by the plasma column. Figure 2 presents the eigenmode propagation constants $p_{m, n}$ normalized to the free-space wave number $k_{0}$ as functions of frequency for a few modes with the azimuthal index $m=1$. The dashed line in Fig. 2 shows the frequency dependence of the normalized (to $k_{0}$ ) wave number $P(\omega)=$ $(\varepsilon+|g|)^{1 / 2}$ of the extraordinary wave propagating exactly in the direction of the external magnetic field in a homogeneous magnetoplasma the parameters of which coincide with those inside the column. The ordinary wave of such a plasma is evanescent in the case considered. The number of propagating eigenmodes is infinite since the magnetoplasma in the column is resonant. In Fig. 2, we plotted only 17 dispersion curves for eigenmodes with the radial indices $n=25, \ldots, 41$.

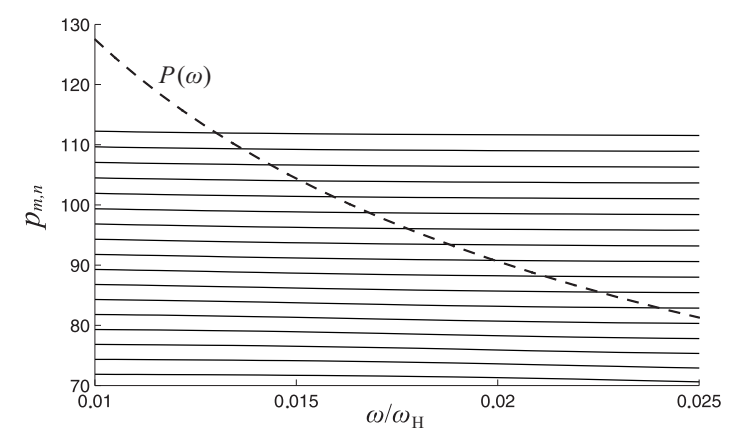

Fig. 2. Dispersion curves of the $m=1$ modes with the radial indices $n=25, \ldots, 41$ (solid lines) and the frequency dependence $P(\omega)$ (dashed line) for $N=10^{13} \mathrm{~cm}^{-3}, B_{0}=800 \mathrm{G}, a=2.5 \mathrm{~cm}$, and $\varepsilon_{a}=1$.

To illustrate the field structures of eigenmodes, Fig. 3 shows the components of the fields $\boldsymbol{E}_{m, n}(\rho)$ and $\boldsymbol{H}_{m, n}(\rho)$ of an eigenmode with the azimuthal index $m=1$ and the radial index $n=18$ as functions of the radial coordinate $\rho$ for the above-mentioned parameters of the column and $\omega / \omega_{\mathrm{H}}=1.21 \times 10^{-2}$. The normalized propagation constant of this eigenmode amounts to $p_{m, n}=52.26$. It is seen that the mode fields has both large- and fine-scale oscillations over the radius. It is interesting to mention that the number of fine-scale oscillations increases with increasing radial index.

Radiation resistances $R_{m}^{\text {mod }}$ corresponding to eigenmodes with fixed azimuthal indices $m$ are presented in Fig. 4 for the above-mentioned antenna and plasma-column parameters. The quantity $R_{m}^{\text {mod }}$ is yielded by summing the partial radiation resistances $R_{m, n}^{\text {mod }}$ over $n$. The radiation resistance $R^{\text {cs }}$ to the surrounding medium turns out to be negligibly small compared with the total radiation resistance to the eigenmodes and, therefore, is not discussed here for brevity.

It is seen in Fig. 4 that the quantities $R_{m}^{\text {mod }}$ decrease with increasing $|m|$, and the main contribution to the radiated power
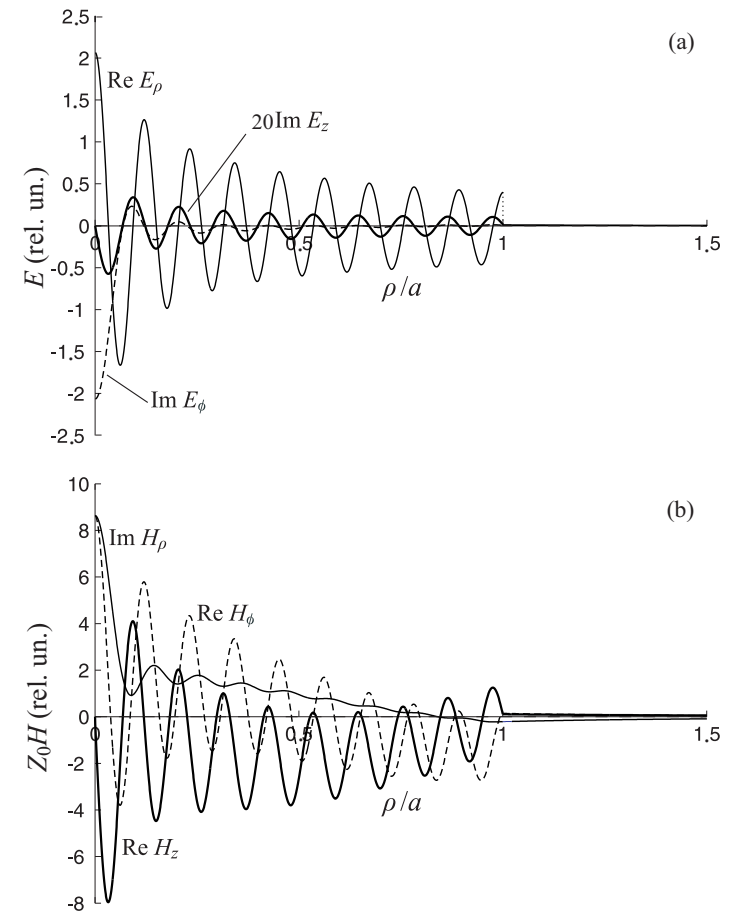

Fig. 3. Components of the (a) electric field $\boldsymbol{E}_{m, n}(\rho)$ and (b) magnetic field $\boldsymbol{H}_{m, n}(\rho)$ of an eigenmode with the indices $m=1$ and $n=18$ as functions of the coordinate $\rho$ for $\omega / \omega_{\mathrm{H}}=1.21 \times 10^{-2}$. Other parameters are the same as in Fig. 2.

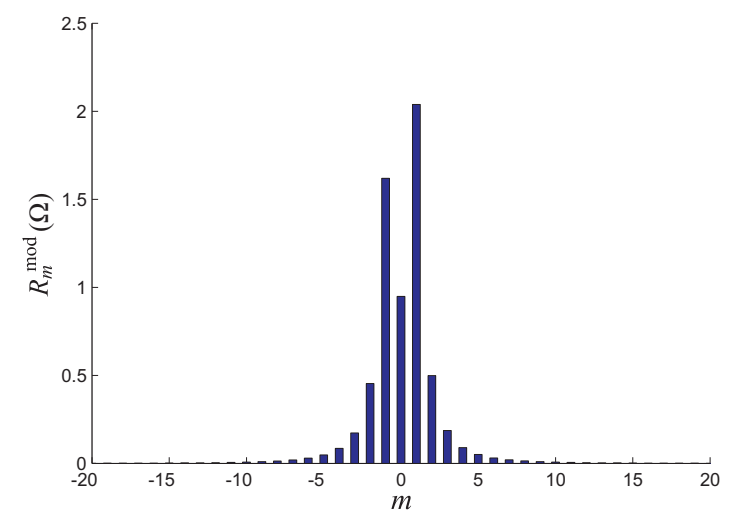

Fig. 4. Radiation resistances $R_{m}^{\text {mod }}$ going to eigenmodes as functions of the azimuthal index $m$ for $a=2.5 \mathrm{~cm}, d / a=0.02, \Delta=0.05 \mathrm{rad}, \phi_{0}=0$, $\varepsilon_{a}=1, N=10^{13} \mathrm{~cm}^{-3}, B_{0}=800 \mathrm{G}$, and $\omega=1.7 \times 10^{8} \mathrm{~s}^{-1}$.

comes from the modes with the azimuthal indices $m= \pm 1$ and $m=0$, which are excited by the azimuthal harmonics of the antenna current with the same indices $m$.

It is interesting that the distribution in Fig. 4 demonstrates asymmetry about the index $m=0$. This asymmetry is stipulated by the gyrotropy of the plasma inside the column. The reversal of the direction of the external magnetic field results in that the distribution in this figure changes to the distribution yielded by the replacement $R_{m}^{\bmod } \rightarrow R_{-m}^{\bmod }$.

Figures 5 and 6 show the partial radiation resistances $R_{m, n}^{\text {mod }}$ corresponding to eigenmodes with the radial indices $n=1,2, \ldots$ and fixed azimuthal indices $m=0$ and $m=1$, 
respectively.

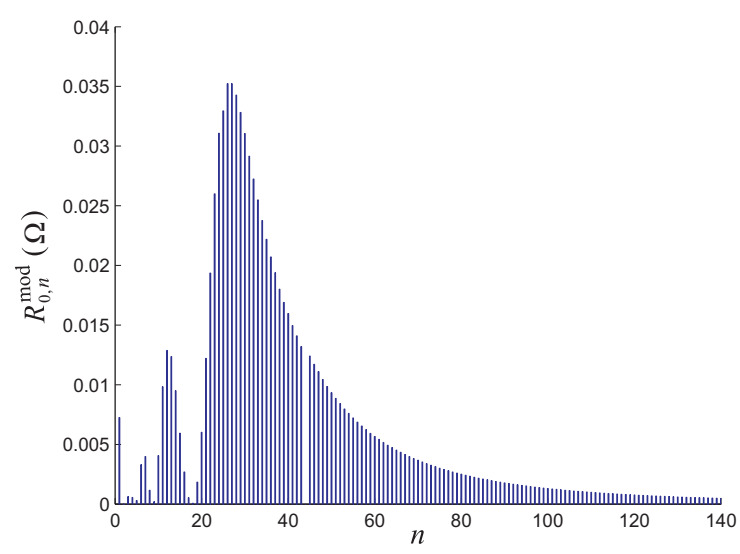

Fig. 5. Partial radiation resistances $R_{m, n}^{\mathrm{mod}}$ to eigenmodes with the azimuthal index $m=0$ and the radial indices $n=1,2, \ldots$. Other parameters are the same as in Fig. 4.

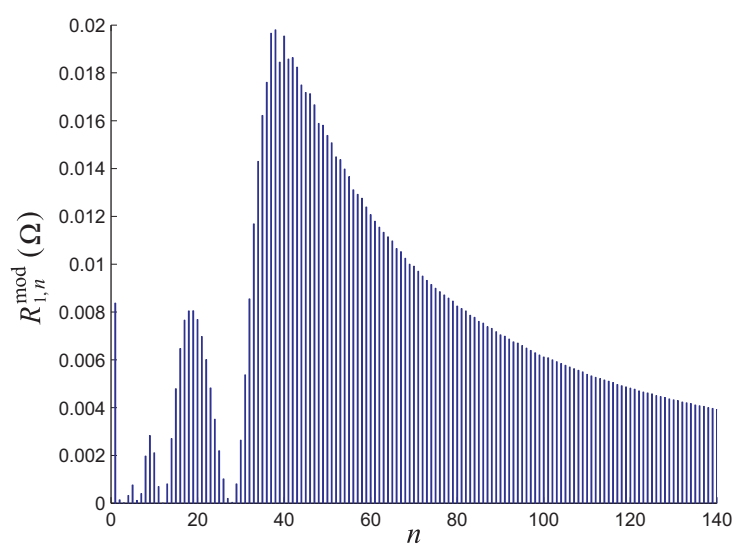

Fig. 6. Partial radiation resistances $R_{m, n}^{\mathrm{mod}}$ to eigenmodes with the azimuthal index $m=1$ and the radial indices $n=1,2, \ldots$. Other parameters are the same as in Fig. 4.

It is seen in Figs. 5 and 6 that the main part of the energy going to the eigenmodes, i.e., discrete-spectrum waves, is spent for excitation of modes with the radial indices up to $n \sim 100$ for $m=0$, and by modes with even greater $n$ for $m \neq 0$. Note that the quantities $R_{m, n}^{\bmod }$ in Figs. 5 and 6 for neighboring modes usually have close values. However, the values of partial radiation resistances for a few eigenmodes significantly differ from the corresponding values for neighboring eigenmodes. This difference appears if the radius of the plasma column becomes close to the minimum or maximum of the azimuthal electric field in the corresponding eigenmode, which results in respective minima or maxima of the eigenmode excitation coefficients.

It can be concluded from the results reported that the power radiated from a loop antenna on the surface of a column filled with a resonant magnetoplasma is predominantly spent for the excitation of the guided modes rather than the radiation field outside the column. This means that such a system is better suitable for increasing the power going to the plasma, which can be important for, e.g., RF plasma sources such as helicon plasma devices (see [4] and references therein).

It should be emphasized that such behavior of the antenna radiation characteristics is solely stipulated by the excitation of an infinite number of propagating eigenmodes by the antenna current in the resonant frequency range of a magnetoplasma. If the plasma inside the column were nonresonant, for which $\operatorname{sgn} \varepsilon=\operatorname{sgn} \eta$, then a situation would be possible where $R^{\mathrm{cs}}>$ $R^{\text {mod }}$ under certain conditions [5]. Such a case is evidently more suitable for the excitation of the radiation outside the plasma column.

\section{CONCLUSION}

In this paper, we analyzed the radiation from a loop antenna located on the surface of an axially magnetized plasma column and operated in the resonant frequency range of a magnetoplasma. The radiation characteristics were calculated for the antenna current found as a result of the rigorous solution of the integral equations for this current. The results obtained make it possible to clarify the distribution of the power radiated from such an antenna over the spatial spectrum of the excited waves. An important implication following from this analysis is that the power radiated from a loop antenna on the surface of a column filled with a resonant magnetoplasma almost entirely goes to eigenmodes which are guided by such a column and include quasielectrostatic modes. It may also be noted that similar behavior will be observed in the case where the column, instead of a magnetoplasma, is filled with a resonant (hyperbolic) anisotropic metamaterial, because such a column is also capable of supporting an infinite number of propagating eigenmodes including those which possess quasielectrostatic properties.

\section{ACKNOWLEDGMENT}

This work was supported by the Government of the Russian Federation (contract No. 11.G34.31.0048), the Russian Foundation for Basic Research (project Nos. 12-02-00747-a and 14-01-31280), and the Grant of the President of the Russian Federation (project No. MK-4688.2014.2).

\section{REFERENCES}

[1] A. V. Kudrin, E. Yu. Petrov, and T. M. Zaboronkova "Current distribution and input impedance of a loop antenna in a cold magnetoplasma," $J$. Electromagn. Waves Appl., vol. 15, no. 3, pp. 345-378, March 2001.

[2] I. G. Kondrat'ev, A. V. Kudrin, and T. M. Zaboronkova, Electrodynamics of Density Ducts in Magnetized Plasmas, Amsterdam, The Netherlands: Gordon and Breach, 1999.

[3] A. V. Kudrin, A. S. Zaitseva, T. M. Zaboronkova, and S. S. Zilitinkevich "Current distribution and input impedance of a strip loop antenna located on the surface of a circular column filled with a resonant magnetoplasma," PIER B, vol. 55, pp. 241-256, October 2013.

[4] A. V. Kudrin, V. A. Es'kin, C. Krafft, and T. M. Zaboronkova, "Whistler wave excitation by a loop antenna in a bounded collisional magnetoplasma," Phys. Scr., vol. 77, no. 5, p. 055501, May 2008.

[5] A. V. Kudrin, A. S. Zaitseva, T. M. Zaboronkova, C. Krafft, and G. A. Kyriacou, "Theory of a strip loop antenna located on the surface of an axially magnetized plasma column," PIER B, vol. 51, pp. 221-246, March 2013. 